\title{
A comparison of digitized frozen section and smear preparations for intraoperative neurotelepathology
}

\author{
Peter V. Gould* and Stephan Saikali \\ Service d'Anatomopathologie et de Cytologie, Centre Hospitalier Affilié Universitaire de Québec, \\ Hôpital de l'Enfant-Jésus, Québec, Canada
}

\begin{abstract}
Background: Intraoperative consultations in neuropathology are often assessed by smear preparations rather than by frozen sections. Both techniques are standard practice for light microscopic examination on site, but there is little data comparing these techniques in a telepathology setting.

Methods: Thirty cases of brain tumours submitted for intraoperative consultation at our institution between July and December 2010 were identified in which both frozen section and tissue smear preparations were available for digitization at $20 \times$ magnification. Slides were digitized using a Hamamatsu Nanozoomer 2.0 HT whole slide scanner, and resulting digital images were visualized at $1680 \times 1050$ pixel resolution with NDP. view software.

Results: The original intraoperative diagnosis was concordant with the sign out diagnosis in 29/30 cases; one tumeur was initially interpreted as a high grade glioma but proved to be a lymphoma at sign out.

Digitized frozen section slides were sufficient for diagnosis at $10 \times$ magnification in 27/30 cases. Digitized tissue smears were sufficient for diagnosis at $10 \times$ magnification in $28 / 30$ cases. In two cases tumour was present on the tissue smear but not the frozen section (one case of recurrent astrocytoma, one case of meningeal carcinomatosis). In one case of lymphoma, tumour was present on frozen section only. These discrepancies were attributed to tissue sampling rather than image quality.

Examination of digitized slides at higher magnfication $(20 \times)$ permitted confirmation of mitoses and Rosenthal fibers on tissue smear preparations, but did not change the primary diagnosis. Intra-slide variations in tissue thickness on smear preparations led to variable loss of focus in digitized images, but did not affect image quality in thinner areas of the smear or impede diagnosis.

Conclusion: Digitized tissue smears are suitable for intraoperative neurotelepathology and provide comparable information to digitized frozen sections at medium power magnification.
\end{abstract}

Keywords: Telepathology, brain tumor, frozen section, cytological smear

\section{Introduction}

Intraoperative assessment of neurosurgical specimens is a common use of telepathology systems, permitting expert analysis of tissue samples without a neuropathologist on site. The University of Pittsburg Medical Center introduced such a system in 2002 [1] but the first studies to examine the feasibility of remote video microscopy in this context were published almost 10 years earlier [2]. Subsequent studies in

\footnotetext{
${ }^{*}$ Corresponding author: Peter V. Gould, Service d'Anatomopathologie et de Cytologie, Centre Hospitalier Affilié Universitaire de Québec, Hôpital de l'Enfant-Jésus, 1401, 18e rue, Québec, QC G1J 1Z4, Canada. E-mail: peter.gould@fmed.ulaval.ca.
}

Europe validated remote imaging techniques for neurotelepathology (also referred to as teleneuropathology by some authors) using standard four micron sections of formalin-fixed, paraffin-embedded tissue stained with hematoxylin and eosin [3].

In Canada the use of neurotelepathology in routine practice was pioneered by the University Health Network in Toronto, which implemented a telepathology system in 2004 to cover the neurosurgical service at the Toronto Western Hospital without a neuropathologist on site [4].

As neuropathologists interested in remote assessment of neurosurgical biopsy specimens, we naturally wish to use the same techniques that would be used 

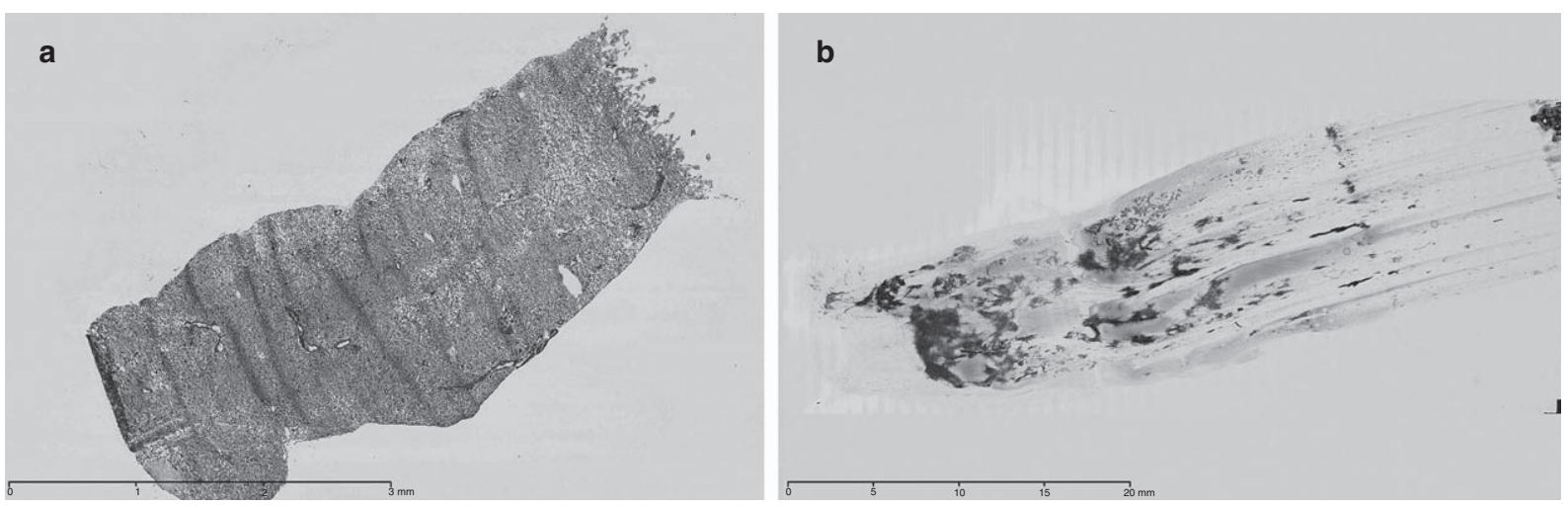

Fig. 1. A) Frozen section - needle biopsy. B) Smear. Pre-op MRI: Single lesion, metastasis vs glioblastoma. Specimen for intraoperative consultation consisted of a single tissue core, $1.1 \times 0.2 \times 0.1 \mathrm{~cm}$. Final diagnosis: glioblastoma.

for on-site assessment. For intraoperative consultations these include cytological smear or squash preparations as well as cryostat frozen sections. Both techniques are commonly used in neuropathology practice, and well described in the literature [5, 6]. Smear preparations are particularly useful for evaluation of small neurosurgical biopsy specimens such as stereotaxic core biopsies (Fig. 1), since they use less tissue than a conventional frozen section and do not introduce freezing artefacts. Since the principal aim of intraoperative consultation in neuropathology is usually to establish the presence of abnormal tissue, to be further characterized on permanent sections, it is desirable to use as little tissue as possible.

Despite the frequent use of smear preparations for intraoperative neuropathologic diagnosis, there is little data in the literature comparing digitized frozen sections and smear preparations. A recent publication from the University Health Network discussed the technical aspects of frozen section diagnosis in the context of teleneuropathology, and included several images of scanned smear preparations, but did not specify whether one technique was superior to the other [7].

In the case of intraoperative smears there are potential limitations to using whole slide virtual images which do not apply to frozen sections. Smear preparations typically include regions of variable thickness which could result in poorly focused single plane images and loss of diagnostic information when compared to the original glass slides for which multiple focal planes in the Z-axis can be examined as needed.

We sought to assess the validity of intraoperative smears for neurotelepathology in the context of the
Eastern Quebec Telepathology network. The CHAUQ Hôpital de l'Enfant-Jésus is recognized by the Programme québécois de lutte contre le cancer as a supraregional (level 4) neuro-oncology centre, covering the territory of the RUIS-Laval, with an active neurosurgical oncology service, and an in hospital neuropathology service. Few neurosurgeons practice elsewhere in the Eastern Quebec territory, therefore the role of neurotelepathology in the Eastern Quebec Telepathology network is at this time a supportive one, permitting after hours and extended coverage when the staff neuropathologists might not be immediately available on site. Whole slide digital imaging was introduced at our hospital in June 2010, and routine neurosurgical intraoperative consultations were processed for digital analysis starting in July 2010.

\section{Methods}

Thirty cases of brain tumours submitted for intraoperative consultation at CHAUQ Hôpital de l'Enfant-Jésus between July and December 2010 were identified in which both frozen section and tissue smear preparations were available for digitization at $20 \times$ magnification.

Slides were digitized using a Hamamatsu Nanozoomer 2.0 HT whole slide scanner, and resulting whole slide digital images in.ndpi format were visualized with NDP.view software (Olympus) on a standard PC with an Intel Core2Quad processor, running Windows XP. Full screen resolution was $1680 \times 1050$ pixels using a flat screen LCD monitor (HP L2245 wg). 
Digital images were assessed for presence or absence of diagnostic lesions, following standard criteria for CNS tumour diagnosis [8].

\section{Results}

A specific pre-operative diagnosis was provided by the neurosurgeon in 5/30 cases, with the remaining cases identified as "tumour" or "space occupying lesion". Additional information including pre-operative neuroradiology was frequently available in the hospital information system to provide a context for intra-operative consultation.

Digitized frozen section slides were sufficient for diagnosis at $10 \times$ magnification in $27 / 30$ cases. Digitized tissue smears were sufficient for diagnosis at $10 \times$ magnification in 28/30 cases. In two cases tumour was present on the tissue smear but not the frozen section, including one case of recurrent astrocytoma and one case of meningeal carcinomatosis. In one case of lymphoma, tumour was present on frozen section only. These discrepancies were attributed to tissue sampling rather than image quality.

Examination of digitized slides at higher magnfication $(20 \times)$ permitted confirmation of mitoses and Rosenthal fibers on tissue smear preparations, but did not change the primary diagnosis. Intra-slide variations in tissue thickness on smear preparations led to variable loss of focus in digitized images, but did not affect image quality in thinner areas of the smear or impede diagnosis.
Three cases were diagnosed as meningioma (Fig. 2), one case as pituitary adenoma, one case as lymphoma, and one case as an inflammatory lesion. One case showed meningeal carcinomatosis (Fig. 3).

Intraoperative diagnosis of tumour type was deferred in five cases. The remaining cases were diagnosed as gliomas, with a diagnosis of high grade glioma in eleven cases (Fig. 4). One case was diagnosed as a low grade glioma with microcalcifications (Fig. 5) and one case as a pilocytic astrocytoma. In one case the tissue sample was interpreted as the edge of a glioma, or brain around tumour (Fig. 6).

The original intraoperative diagnosis was concordant with the sign out diagnosis in 29/30 cases. No case was incorrectly classified as tumour. Three cases identified as glioma were subsequently diagnosed as glioblastoma based on examination of permanent sections, as was the case of brain around tumour.

One case that was initially interpreted as a high grade glioma proved to be a large B-cell lymphoma at sign out (Fig. 7), as confirmed by immunohistochemical staining for CD20. Examination of digitized images from this case confirmed the difficulty of intraoperative diagnosis when the typical perivascular infiltrate of cerebral lymphoma was masked due to widespread tumour infiltration and absence of glial reaction.

Preoperative imaging in this case revealed a $6 \mathrm{~cm}$ lesion of the splenium of the corpus callosum with a second tumour focus at the level of the uncus. The location of the lesion and the pattern of enhancement after gadolinium were strongly suspicious of glioblastoma according to the neuroradiology report. The discrepancy between the intraoperative diagnosis
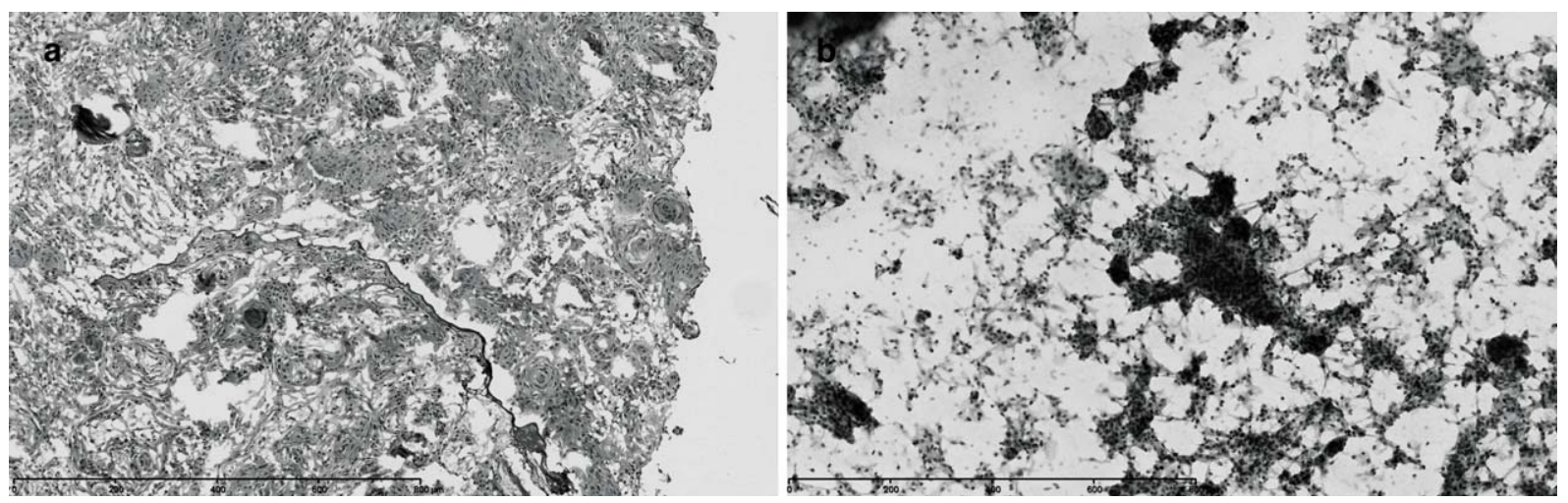

Fig. 2. A) Frozen section - meningioma. B) Smear - meningioma. Pre-op MRI: lesion of optic nerve compatible with meningioma. Specimen for intraoperative consultation consisted of tissue fragments measuring $0.5 \times 0.3 \times 0.1 \mathrm{~cm}$. Final diagnosis: meningioma. 


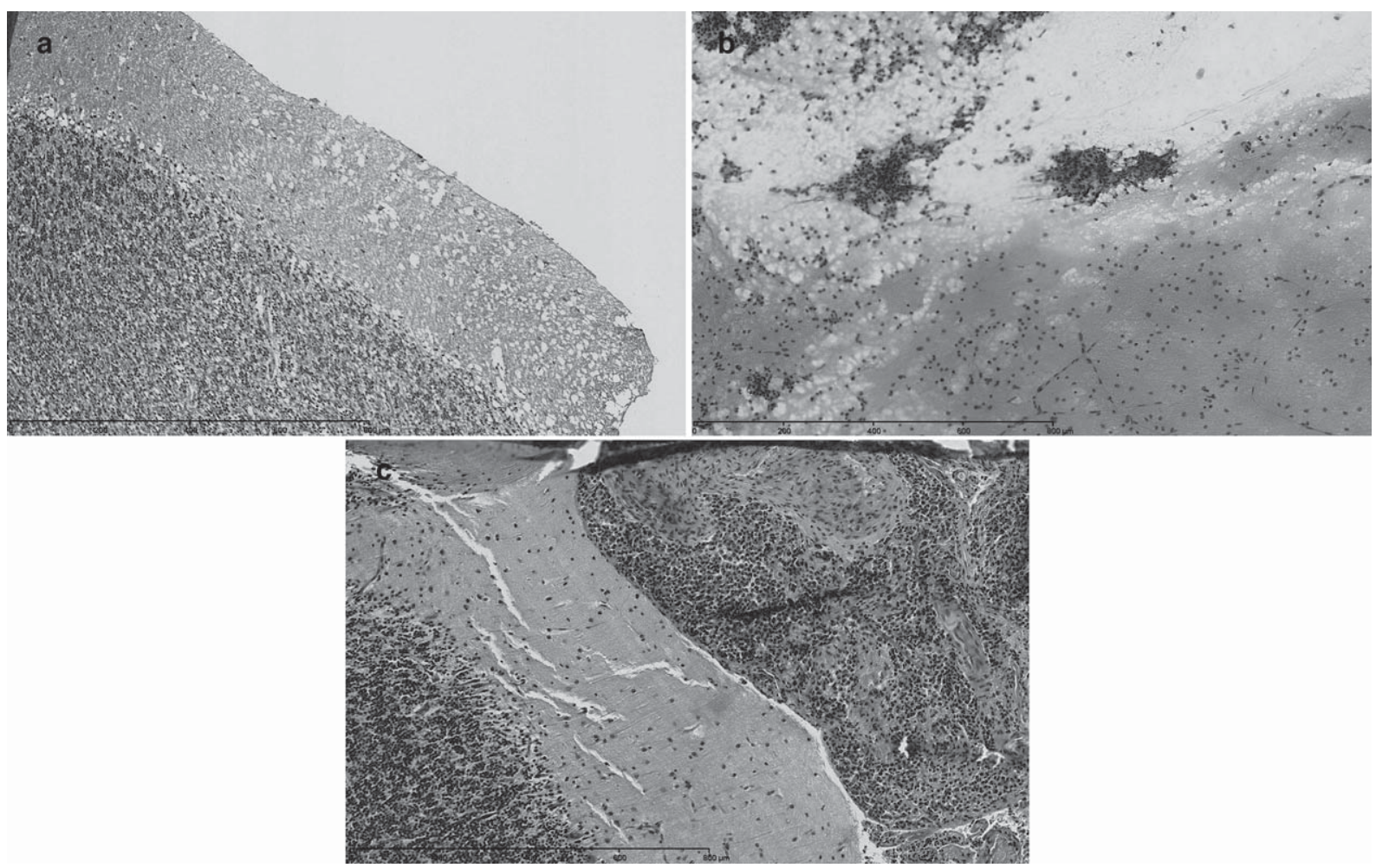

Fig. 3. A) Frozen section - cerebellum. B) Smear - meningeal carcinomatosis. C) Permanent section. Patient with acute onset hydrocephalus. Pre-op MRI: Nodular enhancement: meningeal carcinomatosis favoured over sarcoidosis or infection. Specimen for intraoperative consultation consisted of tissue fragments measuring $0.4 \times 0.2 \times 0.1 \mathrm{~cm}$. Final diagnosis: meningeal carcinomatosis.

and the final diagnosis did not affect patient management.

\section{Discussion}

Previous studies on glass slides have shown that smear preparations are suitable for intraoperative neuropathologic diagnosis $[5,6]$. Our current study shows that they are also suitable for remote diagnosis by telepathology. Reactive astrocytes with abundant cytoplasm and fine filaments were readily identifiable at $10 \times$ magnification, as were malignant glial cells with coarser filaments. Rosenthal fibers were also identifiable at this magnification in the case of pilocytic astrocytoma, and confirmed by re-examination at $20 \times$ magnification. We did not find the thickness of smear preparations to pose an impediment to diagnosis, as many thinner areas of the slide were available for study when searching for glial filaments, and moderate cell overlap in thicker areas did not prevent the identifi- cation of necrotic foci or aberrant vascular structures typical of high grade gliomas.

In keeping with the aim of the original intraoperative consultation, we did not attempt to further classify high grade gliomas into grade III tumours (anaplastic astrocytoma, anaplastic oligodendroglioma, or anaplastic oligoastrocytoma) or grade IV tumours (glioblastoma) based on digital images.

Some limitations of our series should be noted. Our series is retrospective, thus the decision whether or not to make a smear preparation was taken beforehand at the time of intraoperative consultation. Tumours with clear cut pre-operative diagnoses of meningioma or metastasis were generally not sampled for smear preparations during the course of this study. Fibrous tumours such as schwannomas, which are known to be poor candidates for smear preparation, were also not sampled. As a consequence, our series is biased towards adult high grade gliomas, all of which proved to be glioblastomas by routine histology on permanent sections. Due to the small sample size few other CNS tumours types 



Fig. 4. A) Frozen section - high grade glioma. B) Smear - vessels. Diffuse temporale lesion, radiological progression over 15 months. Pre-op MRI: Probable glial tumour with zones of high grade transformation (July 2010). Specimen for intraoperative consultation consisted of two tissue cores, $1.1 \times 0.2 \times 0.1 \mathrm{~cm}$ each. Final diagnosis: glioblastoma. C) Smear - necrosis. Pre-op MRI: Parietal lesion: Glioblastoma. Specimen for intraoperative consultation consisted of tissue fragments measuring $1.0 \times 1.0 \times 0.5 \mathrm{~cm}$. Final diagnosis: glioblastoma.
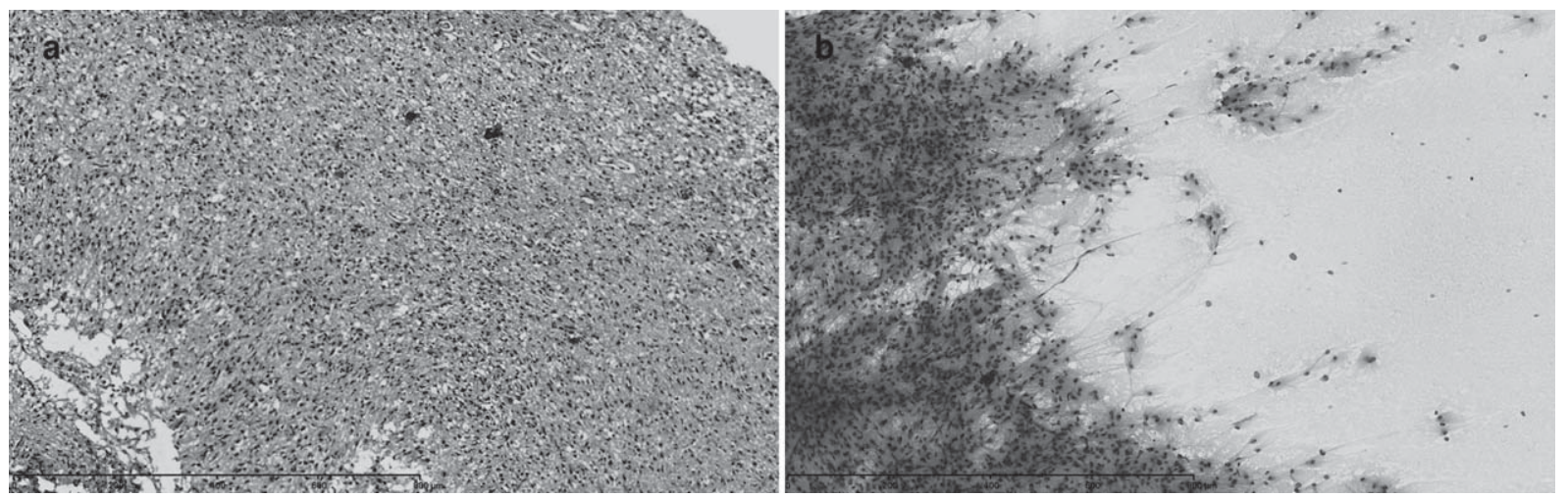

Fig. 5. A) Frozen section - low grade glioma. B) Smear. Pre-op CT: probable low grade glioma with microcalcifications. Pre-op MRI: low grade glioma (oligo) vs subacute ischemia. Specimen for intraoperative consultation consisted of two tissue cores, $1.1 \times 0.2 \times 0.1 \mathrm{~cm}$ each, one of which was used for frozen section and smear preparation. Final histologic diagnosis: Low grade glioma. Analysis of chromosomes $1 \mathrm{p}$ and $19 q$ by FISH showed no evidence of co-deletion. 

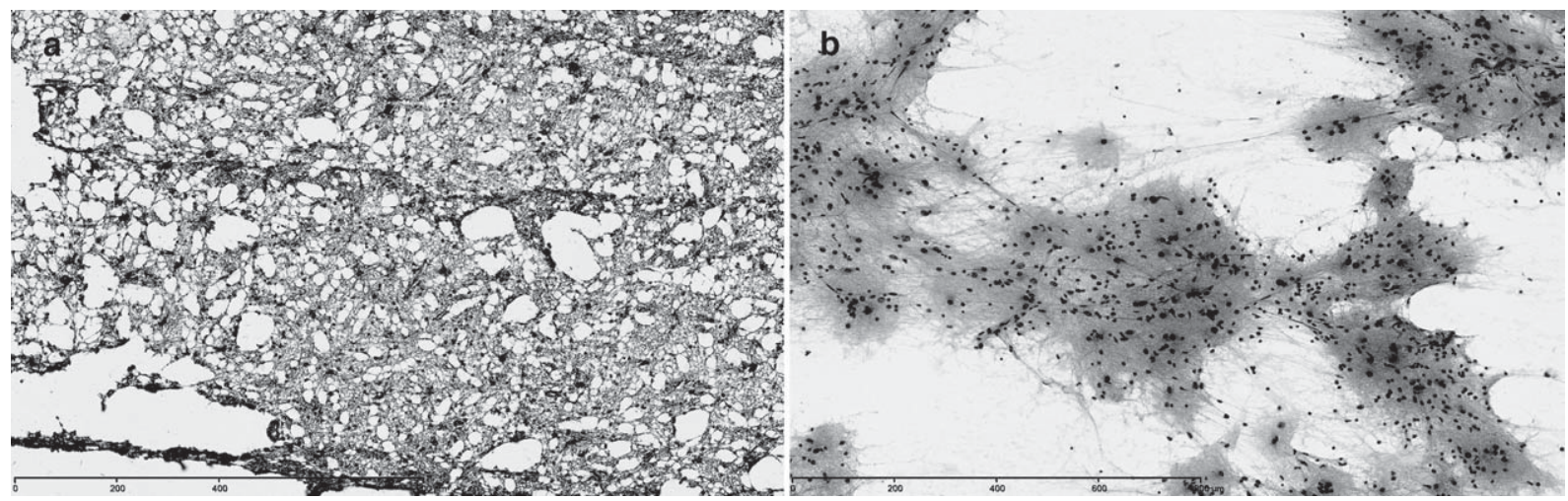

Fig. 6. A) Frozen section - brain around tumour. B) Smear. Pre-op MRI: Two enhancing nodules in the posterior parietal lobe Probable multicentrique GBM. Specimen for intraoperative consultation consisted of tissue fragments measuring $0.5 \times 0.4 \times 0.2 \mathrm{~cm}$. Per-op diagnosis: Compatible with edge of glioma. Specimen for permanent section consisted of tissue fragments measuring $1.0 \times 1.0 \times 0.3 \mathrm{~cm}$. Final diagnosis: glioblastoma.
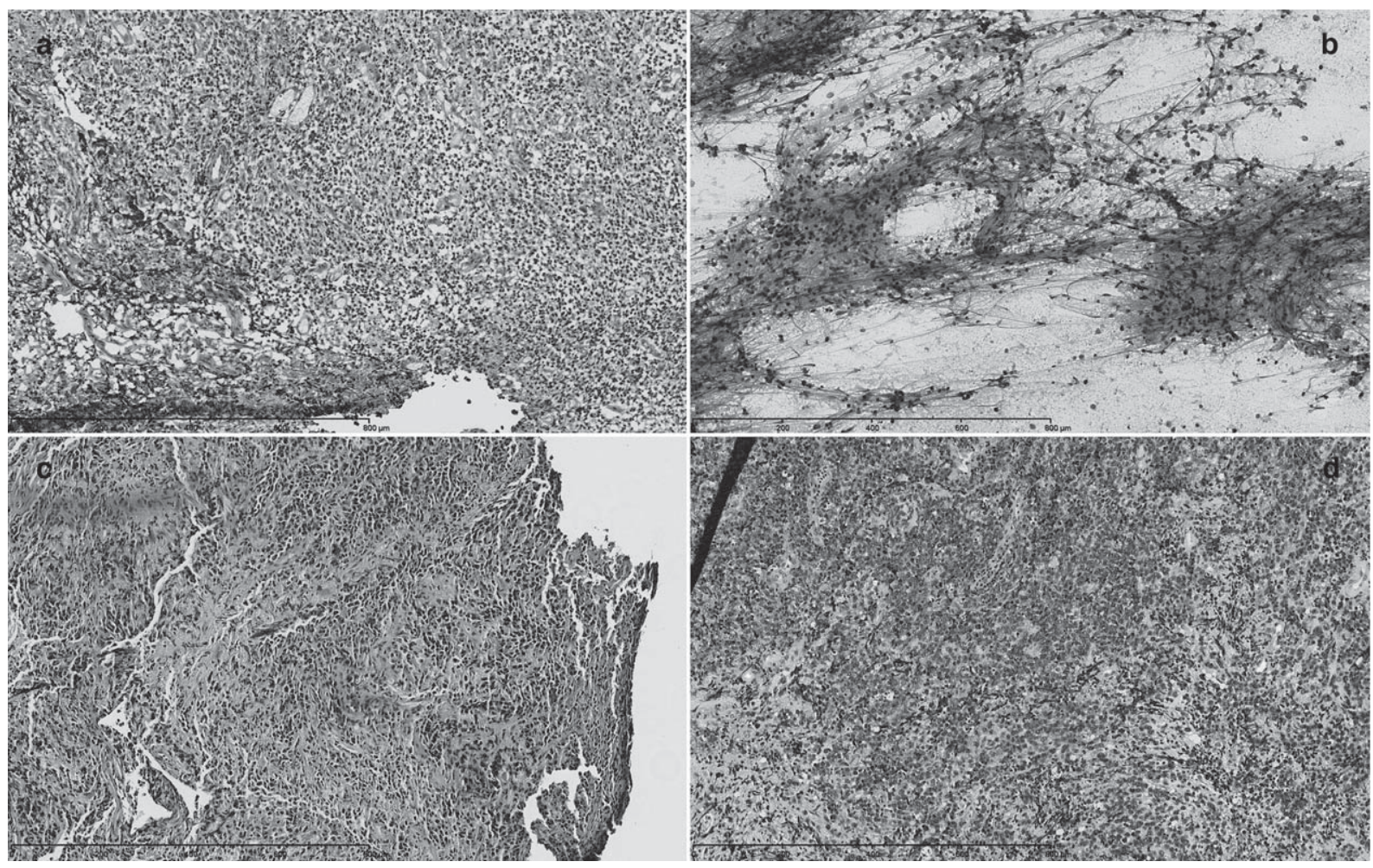

Fig. 7. A) Frozen section - glioma vs lymphoma. B) Smear. C) Permanent A01. D) Permanent B01. Pre-op MRI: $6.1 \mathrm{~cm}$ lesion of the splenium of the corpus callosum with a second tumour focus at the level of the uncus. The location of the lesion and the pattern of enhancement after gadolinium were strongly suspicious of glioblastoma according to the neuroradiology report.Specimen for intraoperative consultation consisted of tissue fragments measuring $0.7 \times 0.2 \times 0.1 \mathrm{~cm}$. Specimen for permanent section consisted of tissue fragments measuring $0.8 \times 0.4 \times 0.3 \mathrm{~cm}$. Final diagnosis: B cell lymphoma, large cell. 
were represented. A larger study would be needed to extend the present findings. The distribution of cases in our series is nevertheless similar to that reported in larger series [9].

Occasional diagnostic discrepancies between the intra-operative and final neuropathological diagnoses such as occurred in our series are recognized in the literature and difficulties in distinguishing diffuse high grade glioma from lymphoma by histology alone are well documented [10]. In our single discordant case the diagnostic pitfalls were numerous, with a mainly solid, partially necrotic lesion composed of small cells that was considered to be characteristic of a glioblastoma on pre-operative imaging, and showed no obvious vasotropism on frozen section or smear preparation that would suggest a histologic diagnosis of cerebral lymphoma. A more conservative intra-operative diagnosis of "small blue cell tumour" would have been appropriate in retrospect but would have been unlikely to affect immediate patient management in view of the radiologic findings.

In conclusion, our study shows that digitized tissue smears are suitable for intraoperative neurotelepathology and provide comparable information to digitized frozen sections at medium power magnification. Our future aim is to expand our series of matched frozen section and smear preparations and to assess the reproducibility of digital image diagnosis between neuropathologists.

\section{References}

[1] C. Horbinski and R.L. Hamilton, Application of telepathology for neuropathologic intraoperative consultations, Brain Pathol 19 (2009), 317-322.
[2] R.L. Becker Jr, C.S. Specht, R. Jones, M.E. Rueda-Pedraza and T.J. O'Leary, Use of remote video microscopy (telepathology) as an adjunct to neurosurgical frozen section consultation, Hum Pathol 24 (1993), 909-911.

[3] J. Szymas, G. Wolf, W. Papierz, B. Jarosz and R.S. Weinstein, Online Internet-based robotic telepathology in the diagnosis of neuro-oncology cases: A teleneuropathology feasibility study, Hum Pathol 32 (2001), 1304-1308.

[4] A.J. Evans, R. Chetty, B.A. Clarke, S. Croul, D.M. Ghazarian, T.R. Kiehl, B.P. Ordonez, S. Ilaalagan and S.L. Asa, Primary frozen section diagnosis by robotic microscopy and virtual slide telepathology: The University Health Network experience, Hum Pathol 40 (2009), 1070-1081.

[5] A.J. Martinez, I. Pollack, W.A. Hall and L.D. Lunsford, Touch preparations in the rapid intraoperative diagnosis of central nervous system lesions: A comparison with frozen sections and paraffin-embedded sections, Mod Pathol 1 (1988), 378-384.

[6] S.Z. Powell, Intraoperative consultation, cytologic preparations, and frozen section in the central nervous system, Arch Pathol Lab Med 129 (2005), 1635-1652.

[7] A.J. Evans, T.R. Kiehl and S. Croul, Frequently asked questions concerning the use of whole-slide imaging telepathology for neuropathology frozen sections, Semin Diagn Pathol 27 (2010), 160-166.

[8] D.N. Luis, H. Ohgaki, O.D. Wiestler and W.K. Cavanee. (eds), WHO Classification of Tumours of the Central Nervous System, 4th ed (2007), IARC Press, Lyon, France.

[9] B.A. Kohler, E. Ward, B.J. McCarthy, M.J. Schymura, L.A. Ries, C. Eheman, A. Jemal, R.N. Anderson, U.A. Ajani and B.K. Edwards, Annual report to the nation on the status of cancer, 1975-2007, featuring tumors of the brain and other nervous system, J Natl Cancer Inst 103 (2011), 714-736.

[10] T.P. Plesec and R.A. Prayson, Frozen section discrepancy in the evaluation of central nervous system tumors, Arch Pathol Lab Med 131 (2007),1532-1540. 


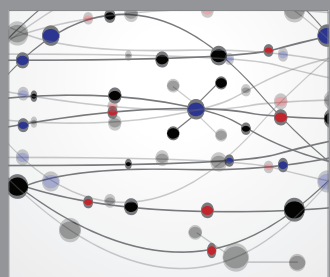

The Scientific World Journal
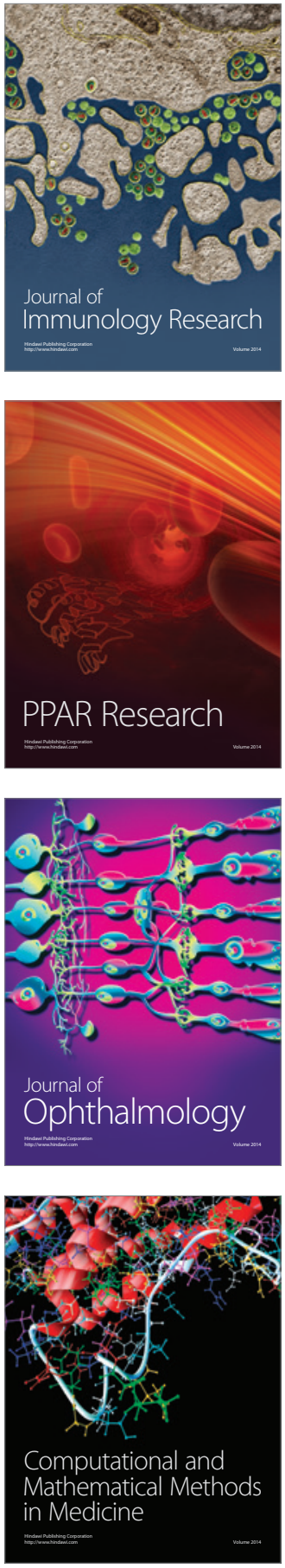

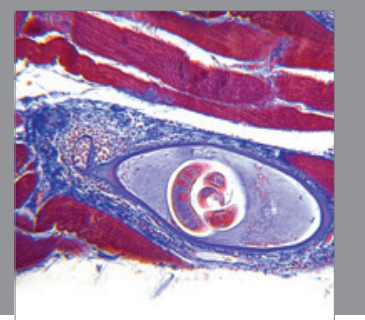

Gastroenterology

Research and Practice
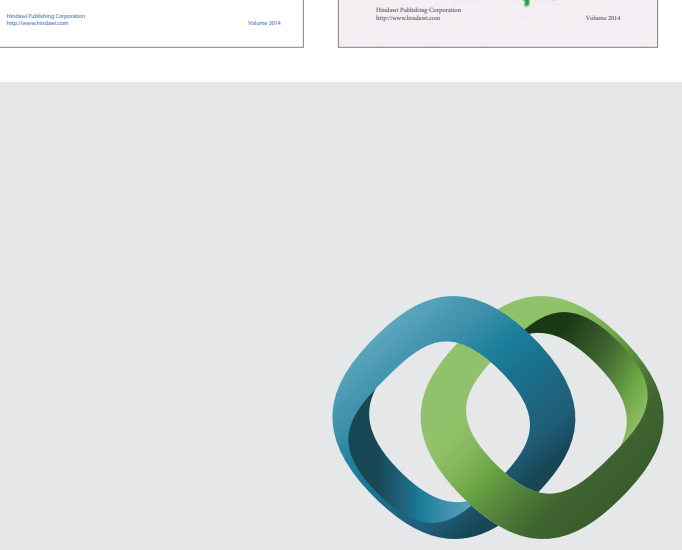

\section{Hindawi}

Submit your manuscripts at

http://www.hindawi.com
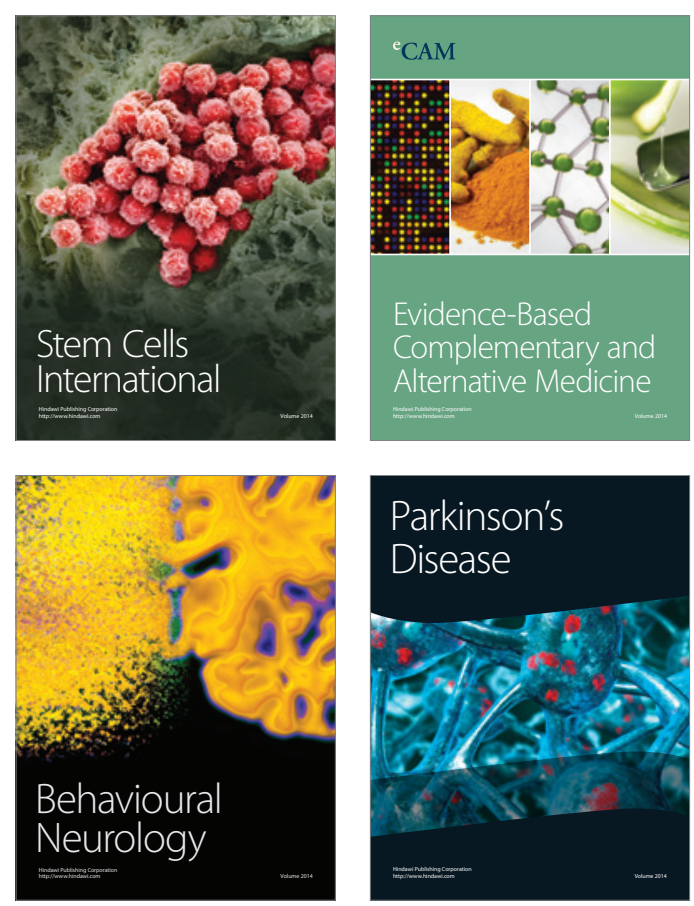

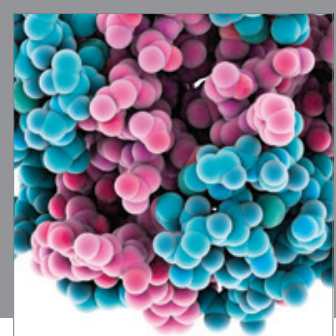

Journal of
Diabetes Research

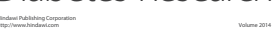



Disease Markers
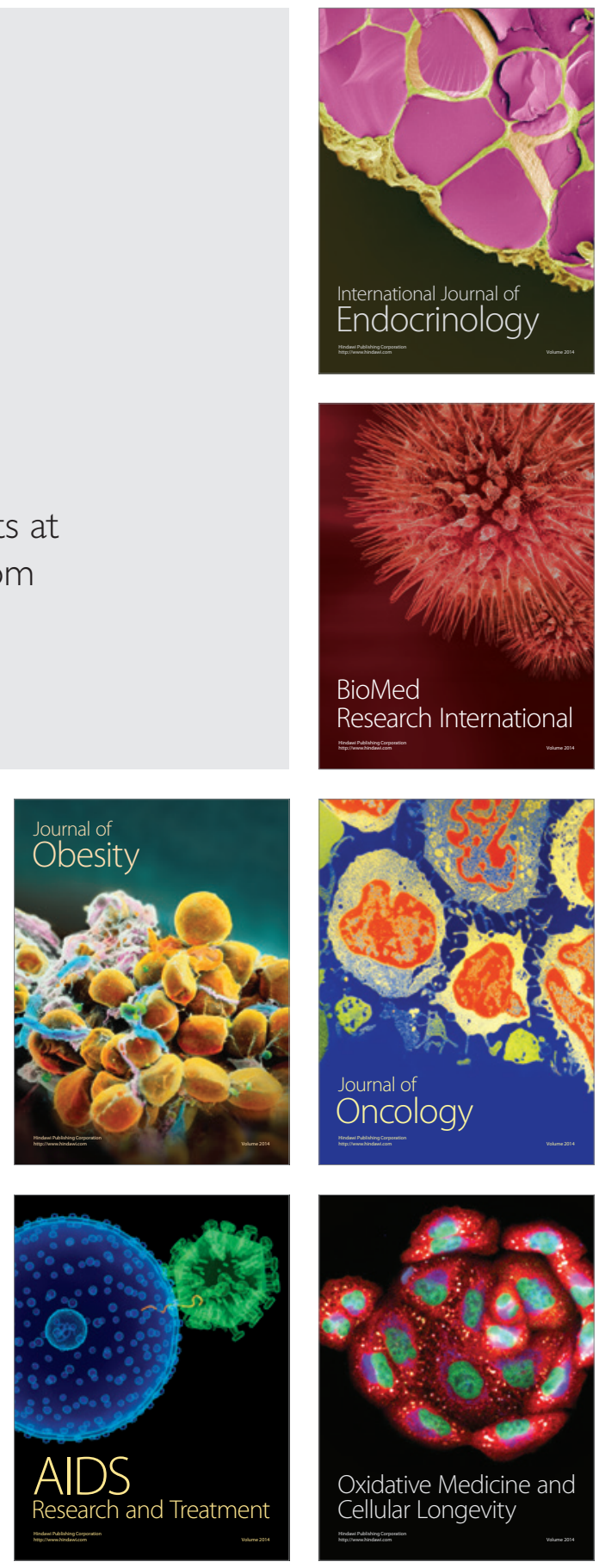